\title{
Identification of diapycnal mixing through optimum multiparameter analysis \\ 2. Evidence for unidirectional diapycnal mixing in the front between North and South Atlantic Central Water
}

\author{
Birgit Klein ${ }^{1}$ \\ Institut für Meereskunde an der Universität, Kiel, Genmany \\ Matthias Tomczak \\ Flinders Institute for Atmospheric and Marine Sciences, Flinders University, Adelaide, South Australia
}

\begin{abstract}
Optimum multiparameter (OMP) analysis is used to analyze mixing in the central water boundary of the tropical North Atlantic Ocean. Diapycnal mixing is found to be prevalent in the frontal region. OMP analysis shows that the mixing is unidirectional (South Atlantic Central Water is always mixed upward into North Atlantic Central Water) but cannot identify the process responsible for the observed diapycnal mixing. A histogram of stability ratios $R_{\rho}$ for all mixing lines shows $R_{\rho}$ values between unity and the value found in the parent water masses. It is suggested that this may indicate competition between isopycnal mixing and double diffusion. Double diffusive fluxes are likely to make a recognizable and significant contribution to diapycnal mixing between the Central Waters.
\end{abstract}

\section{Introduction}

The water masses of the permanent pycnocline, commonly known as Central Water, are characterized by a nearly linear temperature-salinity (TS) relationship. The fact that an unstable salinity gradient is balanced by a stable temperature gradient makes Central Water a suitable environment for salt finger diffusion. It has been proposed [Schmitt, 1981] that the form of the TS diagram in the Central Water is determined by double diffusive processes.

North Atlantic Central Water (NACW) and South Atlantic Central Water (SACW), the water masses of the Atlantic thermocline, are separated by a frontal zone that extends from $20^{\circ} \mathrm{N}$ in the east to about $10^{\circ} \mathrm{N}$ in the west. The front is particularly strong near the African continent where the surface currents are characterized by an upper layer convergence. The currents in the region belong to the subtropical gyre of the North Atlantic Ocean. The Canary Current traverses the region from north to south, feeding cool water from the temperate regions to the tropics. In the vicinity of $20^{\circ} \mathrm{N}$ it veers away from the African shelf to continue as the North Equatorial Current toward the west. This produces cyclonic water movement between the Canary Current and Africa south of $20^{\circ} \mathrm{N}$ (Figure 1) with northward advection of warm water in the east [Tomczak and Godfrey, 1994]. The circulation extends through the upper permanent thermocline. As a result, SACW is advected northward along the African shelf, and the horizontal temperature and salinity gradients

\footnotetext{
${ }^{1}$ Now at Woods Hole Oceanographic Institution, Woods Hole, MA.
}

Copyright 1994 by the American Geophysical Union.

Paper number 94JC01948.

0148-0227/94/94JC-01948\$05.00 between SACW and NACW are enhanced into a water mass front [Zenck et al., 1991]. The front follows the southeastern flank of the Canary Current and is clearly visible in the water mass distribution (Figure 1).

The stratification in both water masses of the Atlantic permanent thermocline is associated with a stability ratio which, over the depth range of their distribution, is nearly constant. The stability ratio $R_{\mathrm{\rho}}$ is defined as

$$
R_{\rho}=\frac{\alpha \Delta \mathrm{T}}{\beta \Delta S}
$$

where $\alpha$ and $\beta$ are the thermal and haline expansion coefficients, respectively, and $\Delta S$ the salinity change over a depth interval where the temperature change is $\Delta T$. Typical values of $R_{\mathrm{\rho}}$ in NACW and SACW are in the range 1.88-1.95.

The regime of nearly constant $R_{\mathrm{\rho}}$ in regions of undisturbed NACW or SACW is in contrast with the situation in the frontal zone between the two water masses in the eastern tropical Atlantic Ocean, which is dominated by intrusions and correspondingly large variations in $R_{\rho}$. Intrusions are produced by interleaving of NACW and SACW across the densitycompensated front. Parcels of NACW penetrate into the SACW environment on isopycnal surfaces and vice versa, forming lenses of unmixed water within the domain of the other water mass. The lenses are eroded and eventually disintegrate as a result of mixing.

It is generally believed that mixing in the oceanic thermocline occurs preferentially on isopycnal surfaces. Lenses and intrusions, which increase the area of contact between water masses with large temperature and salinity differences, are thought to enhance the possibility for diapycnal mixing [Joyce, 1977]. In the present study we present evidence for diapycnal mixing between NACW and SACW in the frontal zone east of $30^{\circ} \mathrm{W}$. Assuming that water properties in the 


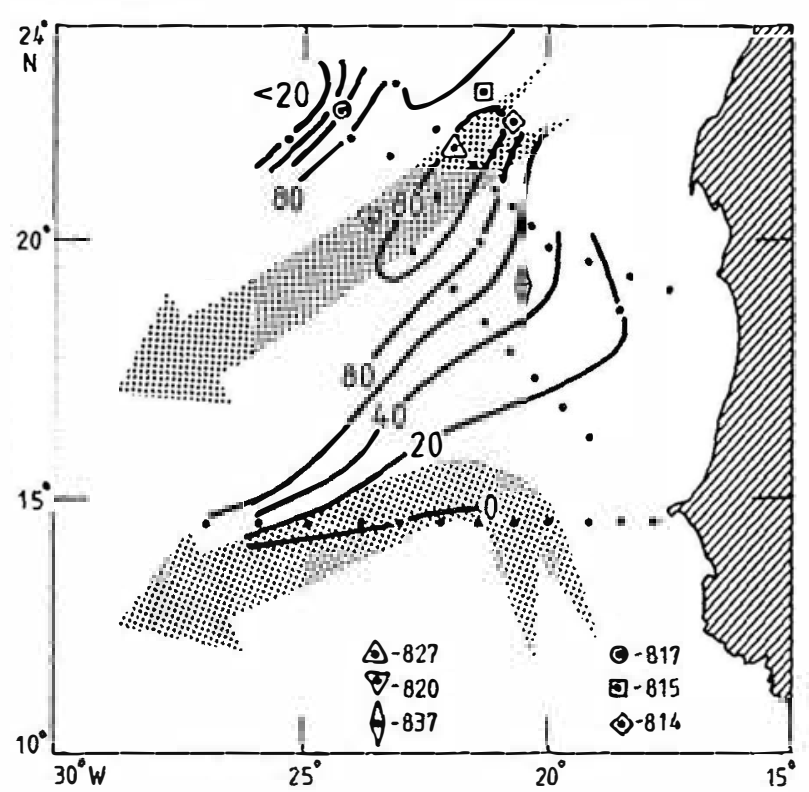

Figure 1. The oceanographic setting in the investigation area, with station positions for FS Meteor cruise 6, November 15 - 27, 1987, and distribution of North Atlantic Central Water (NACW) (water mass content at the 150 dbar level) during the cruise.

frontal zone are the result of a series of mixing events, we determine the property combinations which the source water masses had at the beginning of the mixing. We discuss possible mechanisms responsible for the observed diapycnal mixing and offer reasons to suggest that double diffusion is the most likely process.

The tracing back to the unmixed NACW and SACW requires the use of oxygen and nutrient observations, in addition to observations of temperature and salinity. It will be seen that these water properties which are not responsible for the diapycnal mixing process are, nevertheless, essential for estimating its role in frontal mixing.

\section{Data and Methods}

The data for this study were collected between November 15 and 27, 1987, as part of cruise 6 of FS Meteor. Temperature and salinity were determined using a Neil Brown MK III conductivity-temperature-depth (CTD) system and processed as described by Miiller et al., [1988]. Oxygen and nutrient data were obtained from water samples collected with a General Oceanics rosette sampler.

The analysis of the mixing processes in the frontal zone is based on optimum multiparameter (OMP) analysis as described by Tomczak and Large [1989]. The method represents the source water masses by a limited number of source water types and an associated set of standard deviations, and the mixing between source water masses is analyzed in terms of contributions from the water types (see Tomiczak and Large [1989] and Tomczak et al. [this issue] for details). In the investigation area all property-property relationships for SACW can be approximated by a straight line, so two water types are sufficient to represent SACW. Property-property relationships for NACW show some degree of curvature and cannot be taken as linear over the entire thermocline. To accommodate this, we used three water types to represent NACW (compare Table 1 and Figure 4 below). Six parameters, temperature, salinity, oxygen, phosphate, nitrate, and silicate, are used in the present application of OMP analysis.

Figure 1 shows the positions of the stations occupied by FS Meteor. The frontal zone crosses the investigation area from northeast to southwest. The $50 \%$ contour of NACW or
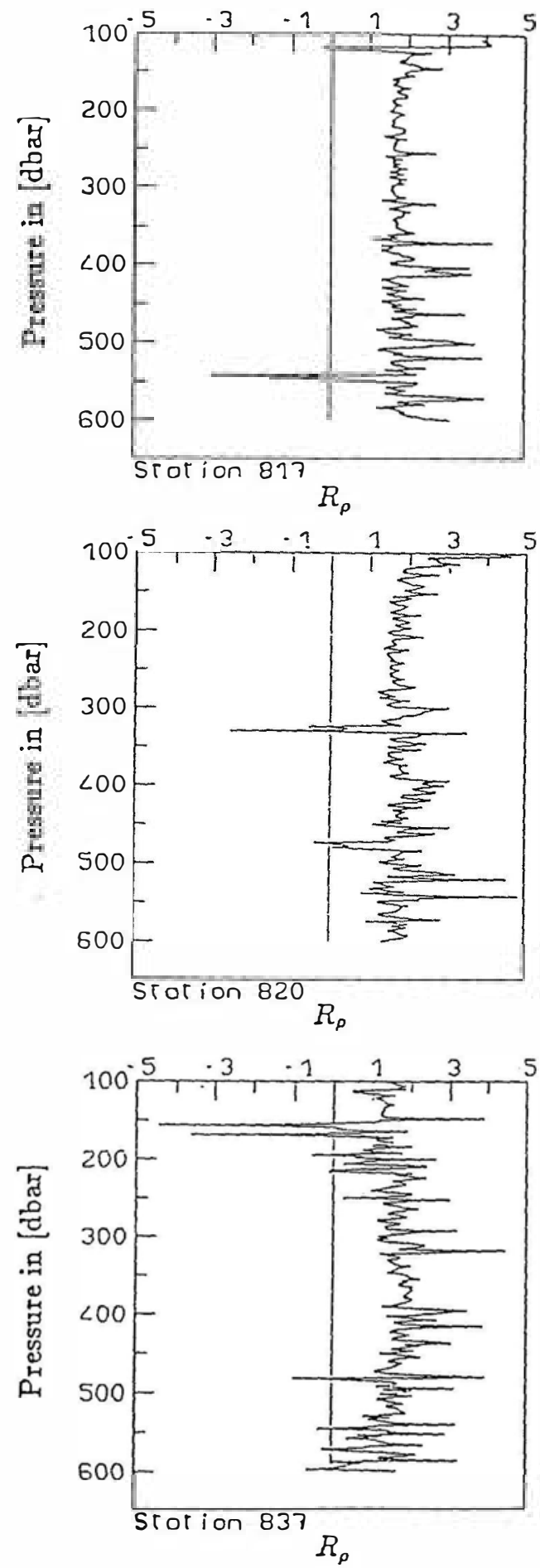

Figure 2. Stability ratio $R_{\rho}$ as a function of depth in (top) North Atlantic Central Water (NACW) (station 817), South Atlantic Central Water (SACW) (station 837) and (bottom) in the frontal zone (station 820), calculated from conductivitytemperature-depth CTD data. 


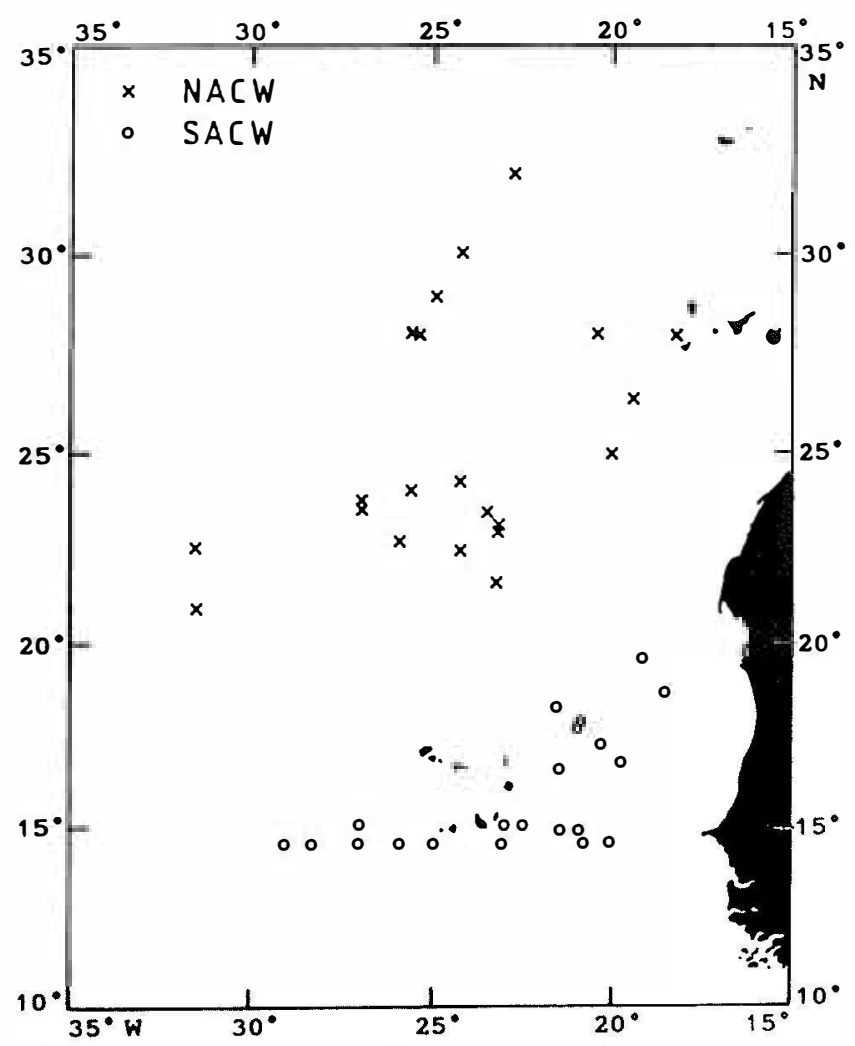

Figure 3. Location of stations used to determine source properties for NACW and SACW.

SACW, which can be taken as the center of the front, is located near $20^{\circ} \mathrm{W}$ on the $150 \mathrm{dbar}$ level. Owing to a frontal meander or an isolated lens with SACW characteristics, the $50 \%$ contour occurs a second time in the northwestern part of the investigation area near $25^{\circ} \mathrm{W}, 22^{\circ} \mathrm{N}$.

The stability ratio $R_{\rho}$ for three stations, calculated from CTD data using central differences over 4 dbar intervals, is shown in Figure 2. Stations 817 and 837 are fairly representative for the situation of NACW and SACW, respectively, below $200 \mathrm{~m}$ depth (i.e., deeper than the depth shown in Figure 1). Both show small $R_{\mathrm{p}}$ variability outside the mixing region. If the data are sent through a low-pass filter (as is often done in studies of water mass stability), then $R_{\mathrm{p}}$ in the NACW is virtually constant over the depth range $200-500 \mathrm{~m}$, while in the SACW, $R_{\mathrm{\rho}}$ varies slightly with depth. Variability is higher in the SACW than in the NACW, possibly as a consequence of enhanced mixing in the SACW during its way through the equatorial current system. Spikes in the $R_{\rho}$ distribution above $200 \mathrm{~m}$ and below $500 \mathrm{~m}$ indicate the limit of the depth range of nearly uniform $R_{\mathrm{p}}$ values; below $600 \mathrm{~m}$, $R_{\rho}$ values fall off rapidly, indicating increasing influence of Antarctic Intermediate Water. Compared with the situation outside the front, vertical profiles of $R_{\mathrm{p}}$ in the frontal zone show a combination of the low-passed trends found in NACW and in SACW, interspersed with intrusions that can occur anywhere in the thermocline. Station 820 (Figure 2) shows such intrusions at 320 and 480 dbar.

Twenty stations north of the frontal zone which contained nearly exclusively NACW were used to determine the properties of the NACW source water types. The stations were from cruise 6 of FS Meteor, augmented by stations from a previous and a later cruise in the same investigation area. Twenty-four stations in the south were used for the derivation of SACW source water types (Figure 3). The data from both sets of stations were subjected to least squares linear fits, which produced linear approximations and standard deviations for all property-property relationships. The source water type definitions were then taken from the points of the linear fits that correspond to the upper and lower end of the depth range studied. To accommodate the deviation of NACW propertyproperty relationships from straight lines, the NACW data were split into two subsets, one for the upper thermocline and one for the lower thermocline, and the procedure was applied to both subsets. Parameter weights were calculated as by Tomczak and Large [1989] and rounded to one decimal place. Table 1 lists source water types, standard deviations, and weights.

OMP analysis is based on the assumption of identical exchange coefficients for all parameters. The question arises whether it can give insight into water mass mixing in a situation where double diffusive processes can be expected to contribute to the distribution of water mass properties. Qualitatively, OMP analysis can identify the presence of diapycnal mixing, including double diffusion. Quantitatively, it is clear that salt fingers are the result of different exchange coefficients for temperature and salinity, and OMP analysis will produce erroneous mixing ratios when applied to a study of mixing on the salt finger scale. Hydrographic station data based on rosette sampling do not resolve the salt-finger scale but provide information on the distribution of water properties on the eddy and intrusion scale (as defined by Joyce, [1977]). OMP analysis will produce a correct description of the water mass distribution on that scale, as long as the small-scale,

Table 1. Source Water Types, Standard Deviations and Weights for North Atlantic and South Atlantic Central Water

\begin{tabular}{|c|c|c|c|c|c|c|c|}
\hline \multirow[b]{2}{*}{ Parameter } & \multicolumn{3}{|c|}{ NACW } & \multicolumn{2}{|c|}{ SACW } & \multirow[b]{2}{*}{$\begin{array}{c}\text { Standard } \\
\text { Deviation }\end{array}$} & \multirow[b]{2}{*}{ Weight } \\
\hline & Upper & Middle & Lower & Upper & Lower & & \\
\hline$\Theta\left({ }^{\circ} \mathrm{C}\right)$ & 18.972 & 13.893 & 10.975 & 15.835 & 8.862 & 0.239 & 1.0 \\
\hline$S$ & 36.800 & 35.880 & 35.498 & 35.805 & 35.035 & 0.034 & 1.0 \\
\hline $\mathrm{NO}_{3}(\mu \mathrm{g}-\mathrm{at} / \mathrm{l})$ & 1.819 & 12.610 & 20.196 & 22.833 & 35.499 & 2.170 & 0.1 \\
\hline $\mathrm{PO}_{4}(\mu \mathrm{g}-\mathrm{at} / \mathrm{l})$ & 0.094 & 0.750 & 1.294 & 1.394 & 2.319 & 0.130 & 0.1 \\
\hline $\mathrm{SiO}_{4}(\mu \mathrm{g}-\mathrm{at} / \mathrm{l})$ & 0.439 & 3.855 & 8.590 & 6.850 & 18.027 & 1.540 & 0.1 \\
\hline $\mathrm{O}_{2}(\mathrm{ml} / \mathrm{l})$ & 4.776 & 4.117 & 3.598 & 1.586 & 1.171 & 0.410 & 0.1 \\
\hline
\end{tabular}

Abbreviations are NACW, North Atlantic Central Water and SACW, South Atlantic Central Water.

See text for description of parameters. 
double diffusive processes do not invalidate its assumption of identical exchange coefficients on the larger eddy and intrusion scale or, to state the same condition in other words, as long as the effect of double diffusive mixing can still be adequately described by straight lines in property-property diagrams derived from rosette sample data. Schmitt [1981] argues that the effect of salt fingering on the distribution of water properties in the oceanic thermocline is an adjustment of the TS relationship on the eddy and intrusion scale to constant $R_{p}$. Such TS relationships deviate slightly from straight lines. The question of the applicability of OMP analysis in the presence of double diffusion therefore reduces to the question of whether the difference between a linear TS relationship and a TS relationship defined by constant $R_{\mathrm{p}}$ is significant enough to make the OMP result meaningless.

While there is no doubt that double diffusion tends to produce TS relationships characterized by constant $R_{\mathrm{\rho}}$, it is not clear whether all water masses of the oceanic thermocline are associated with such TS curves. Tomczak and Gu [1987] analyzed the situation in the western equatorial South Pacific Ocean and found that in some regions the TS curves were better described by a linear TS relationship, while in others the constant $R_{\mathrm{\rho}}$ TS relationship provided the better description. Warmus [1988] confirmed their findings using a rigorous statistical method to produce best linear fits and best constant $R_{\mathrm{\rho}}$ fits pointing out, however, that the difference between the two best fits is statistically only marginally significant. A statistical analysis of thermocline TS relationships for the world ocean proved inconclusive; it showed that for most of the TS relationships in the ocean's thermocline the difference between the best linear fit and the best constant $R_{\rho}$ fit is statistically not significant.

Comparison of TS diagrams for SACW and NACW with constant $R_{\rho}$ curves (Figures 2 and 4 ) suggests that NACW might be described better by a constant $R_{\mathrm{\rho}}$ relationship, while the case of SACW is much more ambiguous. As mentioned before, we accommodate the curvature of the TS curve for NACW by using three source water types rather than two, i.e., by approximating the TS curve by two linear relationships over separate TS ranges. The difference between a constant $R_{p}$

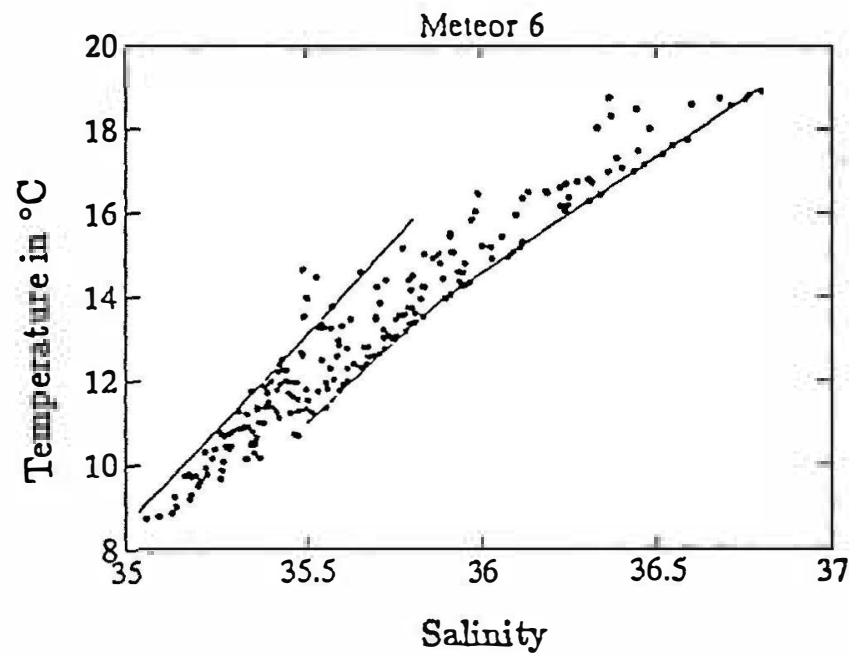

Figure 4. Temperature-salinity (TS) diagram for all stations. The lines show the water mass definitions for NACW and SACW; they connect the TS combinations of the source water types. fit and the chosen linear fits is therefore extremely small. This allows us to apply OMP analysis and discuss mixing lines in TS diagrams in terms of equivalent $R_{\rho}$ values. While such a discussion can only suggest a role for double diffusion in the mixing process and leaves room for debate, it will be seen that the presence of diapycnal mixing in the region is beyond doubt.

Figure 4 compares the source water types with the complete range of parameter values encountered in the observation area during cruise 6 . The temperature-salinity diagram demonstrates that in addition to the "pure" NACW and "pure" SACW (as defined in Table 1 for the purpose of this study), a great variety of mixing products was present in the investigation area. It should be kept in mind that the derived definition lines were computed from data sampled over a 3-year period and during different cruises. The definition curves for NACW and SACW are thus mean curves for a 3-year situation; a few observation points from Meteor cruise 6 fall outside the range spanned by the definition curves as a result of time variability of source water type properties.

\section{Results and Discussion}

Since OMP analysis requires nutrient and oxygen information, it can only be performed at a few depth levels where data from the rosette are available. Typically, between four and eight observations fell within the depth range 200-500 $\mathrm{m}$. Seven equations (six parameter observations plus mass conservation) with five unknowns (water type contributions) defined the system of linear equations, which was solved independently for each observation point. Figure 5 shows the results from three stations in the frontal zone for all rosette observations in that range. The observation points are at the crossover points between the solid and dashed lines. The solid lines connect the TS points on the source definition lines that, according to OMP analysis, were mixed to create the observed temperature-salinity properties and are compatible with the observed nutrient values. The dashed lines are the isopycnals through the observation points. If mixing occurred strictly on isopycnal surfaces, then the mixing lines from OMP analysis should coincide with the broken lines. Any deviation from the isopycnals is an indication for possible diapycnal mixing.

Before accepting the presence of diapycnal mixing in the region, it is necessary to establish the reliability of the result. Tonczak et al. [this issue] establish a threshhold which has to be met before OMP analysis can reliably identify the effects of diapycnal mixing events. They show that in synthetic data designed to simulate the eastern Indian Ocean, diapycnal mixing can be diagnosed if the mean difference between source water type contributions (WTC), determined through OMP and isopycnal analysis over about 120 data points, exceeds $8 \%$ WTC and the mean standard deviation, 15\%WTC. The threshhold is determined by the amount of environmental variability in the source water regions. A quantitative assessment of environmental variability in the source regions of NACW and SACW can only be achieved through an analysis of a large amount of historical data. On a qualitative level a comparison of the hydrographic settings in the two oceans suggests that its effect on OMP analysis is smaller in the Atlantic than in the Indian Ocean.

The ability of isopycnal analysis to resolve mixing is directly proportional to the distance along isopycnals between the source water TS relationships. The TS curves of 


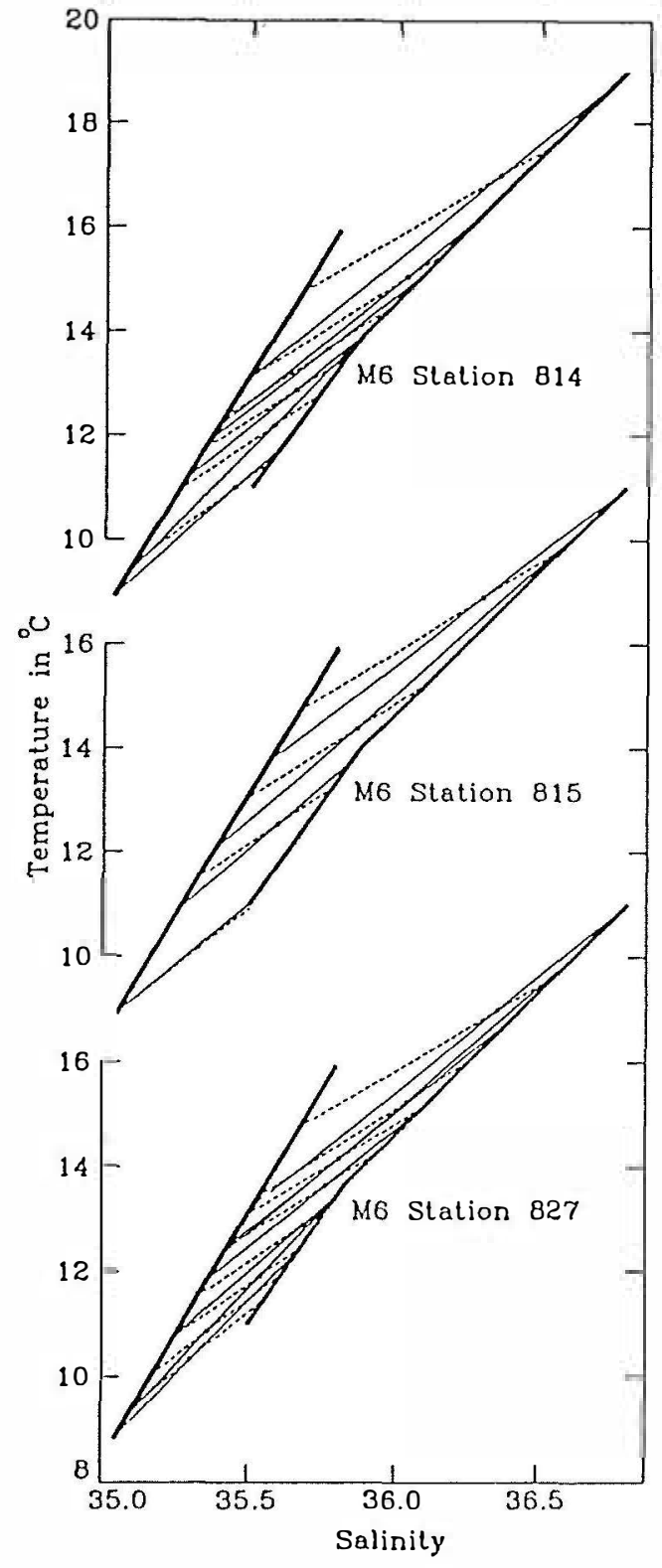

Figure 5. An example of the result from OMP analysis in the frontal zone (stations 814, 815, and 827). Bold, solid lines to the left and right are water mass definition lines. Dashed lines through observation points are isopycnals; solid lines represent the mixing lines determined by OMP analysis.

NACW and SACW are farther apart than the TS curves of the two water masses in the Indian Ocean thermocline, which merge at the lower end of their TS ranges. The same amount of environmental variability (scatter of TS points in the source water masses around their TS relationships) therefore produces smaller departures of calculated values from true values in the Atlantic than in the Indian Ocean situation. In other words, the error introduced into isopycnal analysis by environmental variability is smaller, and the Indian Ocean threshholds of $8 \%$ WTC for the mean difference and $15 \%$ WTC for the standard deviation should be conservative estimates for the significance of differences between OMP and isopycnal analysis in the Atlantic Ocean; Tomczak et al. [this issue] estimate that the respective values are only half those found in the Indian
Ocean. It is evident from Figure 5 that even the Indian Ocean threshhold is exceeded on many occasions within the front. At station 815 , for example, mixing is isopycnal at the lowest data point shown but diapycnal at all other observation levels. The mean difference between OMP and isopycnal solutions at station 815 is $10.3 \%$ WTC; individual differences range from 3 to $20 \%$ WTC. Similar situations can be seen at stations 814 and 827.

Conclusive verification of diapycnal mixing requires statistical treatment of data from more than one station. We analyzed the statistics of $R_{\mathrm{p}}$ since the $R_{\mathrm{p}}$ distribution can give an indication of the mechanism responsible for the diapycnal mixing and, as discussed above, over the range of TS curves present the difference between constant $R_{\mathrm{p}}$ fits and linear TS fits is insignificant. Figure 5 indicates that where departure from isopycnal mixing occurs, mixing always follows a mixing line in the TS diagram which is steeper than the corresponding isopycnal but not as steep as the TS curves of the source water masses. This implies a unidirectional mixing process; SACW water is always mixed upward but never downward (path 1 of Figure 6). Mixing across isopycnals as a result of eddy turbulence should not show a directional preference for one of the two possible mixing directions (it should occur along path 2 of Figure 6 as often as it occurs along path 1); it cannot therefore be the dominant mixing process in this situation. Double diffusive mixing, on the other hand, can explain the observations.

Having shown the existence of unidirectional diapycnal mixing, we now offer a few suggestions why double diffusion could be the dominant mixing process in the frontal region. A characteristic feature of the observed unidirectional mixing is that the values of $R_{\mathrm{\rho}}$ which correspond to the observed mixing lines are always larger than unity (the $R_{\mathrm{p}}$ value of the isopycnals) but smaller than 1.9 (the average value for NACW and $\mathrm{SACW}$ ). To quantify this result, we computed the associated $R_{\mathrm{p}}$ values of the OMP solution lines for all stations in the frontal

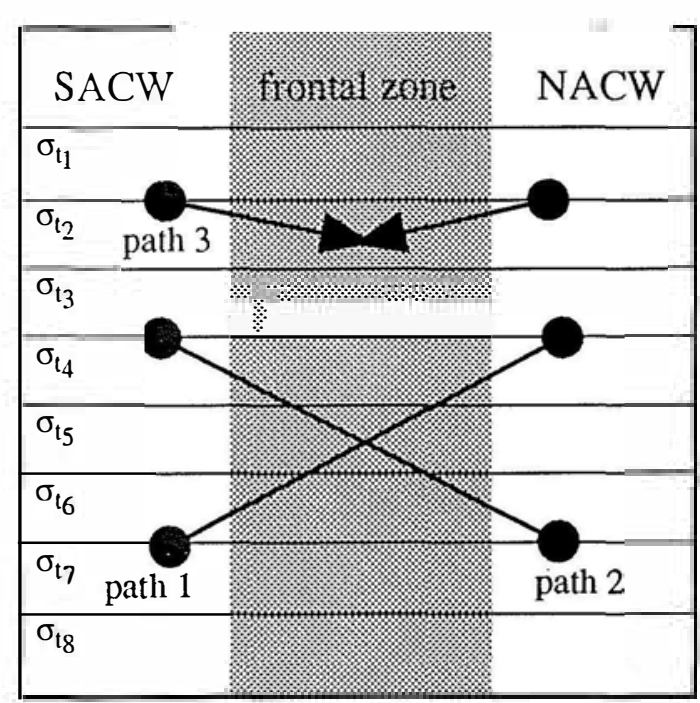

Figure 6. Sketch of mixing directions across the front between SACW and NACW. Path 1 produces stability ratio $R_{\rho}$ values $>1$, path 2 (which is not observed in the field) produces $R_{\rho}$ values $<1$. Turbulent mixing and mixing resulting from shear instability produces mixing along both paths. Cabbeling produces mixing along path 3 . 


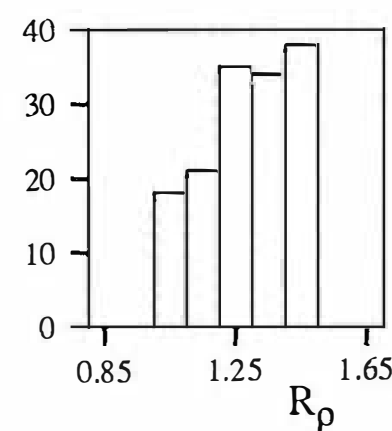

Figure 7. Histogram of stability ratio $R_{\rho}$ of the mixing lines derived from OMP analysis in the frontal zone.

region. Figure 7 shows a histogram of these $R_{\mathrm{p}}$ values. The effect of pressure on the thermal and haline expansion coefficients could not be included in the calculation; the maximum error in $R_{\mathrm{p}}$ caused by this approximation is less than $5 \%$.

The tendency of $R_{\mathrm{p}}$ values to be bound between 1 and 1.5 in the frontal region can be interpreted as the result of two competing processes. Isopycnal mixing events produce local minima of $R_{\mathrm{p}}=1$. Possible processes to act against isopycnal mixing are eddy turbulence, shear-generated turbulence, and cabbeling. Eddies or shear-generated turbulence can cause mixing across isopycnals; but the resulting $R_{\rho}$ distribution should be normally distributed around its isopycnal value. Cabbeling acts unidirectionally, but its effect is much too small to produce the observed departure from isopycnal mixing [Tonczak et al., this issue]. In contrast, double diffusion acts to eliminate local $R_{\mathrm{p}}$ minima and maxima [Schmill, 1981], so that vertical $R_{\rho}$ distributions show little variation across the thermocline outside the frontal zone (Figure 2). In the frontal region, double diffusion attempts to restore the TS curve to a uniform stability ratio. Changing $R_{\mathrm{p}}$ from 1 to values $<1$ would only increase the departure from the original value. Double diffusion therefore can only act to force $R_{p}$ back toward the higher value of the source water masses. This explains the unidirectional character of the mixing in the front.

The growth rate of salt fingers has been evaluated by Schmitt and Evans [1978] who found that the $e$-folding time of finger amplitudes becomes less than the local bouyancy period for $R_{\mathrm{p}}<2$. The $e$-folding times get shorter as $R_{\mathrm{p}}$ approaches 1 . It appears that at the small $R_{\mathrm{p}}$ values found for the mixing events in the frontal zone, salt-fingering processes can become sufficiently strong to avoid disruption by the internal wave field or can be only slightly intermittent.

In conclusion, we have shown the presence of unidirectional diapycnal mixing in the frontal zone between SACW and NACW. We discussed possible mechanisms responsible for the observed diapycnal mixing and identified double diffusion as a likely candidate. It is interesting to note that the existence of diapycnal mixing can be verified by making use of oxygen and nutrients, i.e, tracers which do not determine the mixing processes. Mixing processes of small vertical scale can thus be analyzed from hydrographic bottle data of much coarser vertical resolution.

Acknowledgments. Most of this study was done while one of the authors (M.T.) was on sabbatical leave at the Institut für Meereskunde an der Universität Kiel. Support with facilities is gratefully acknowledged. Comments and suggestions from two reviewers helped us to clarify several points and improve the presentation.

\section{References}

Joyce, T. M., A note on the lateral mixing of water masses, $J$. $P h_{h}$ )'s. Oceanogr. 7, 626-629, 1977.

Müller, T.J., G. Siedler, and W. Zenk, Forschungsschiff Meteor Reise Nr. 6, Atlantik 87/88, Fahrtabschnitte Nr. 1-3, Oktober-Dezember 1987, Ber. Inst. Meeresk., Christian Albrechts Univ., 184, 77 pp.. 1988.

Schmitt, R.W., Form of the temperature-salinity relationship in the Central Water: Evidence for double-diffusive mixing, J. Phys. Oceanogr., 11, 1015-1026, 1981.

Schmitt, R.W., and D. L. Evans, An estimate of vertical mixing due to salt fingers based on observations in the North Atlantic Central Water, J. Geophys. Res., 83, 2913-2919, 1978.

Tomczak, M., and J. S. Godfrey, Regional Oceanograplyy: An Imtr. duction, 348 pp., Pergamon, New York, 1994.

Tomczak, M., and Y. Gu, Water mass properties of the permanen thermocline in the western South Pacific Ocean during WESTROPAC'82., Deep Sea Res., 34, 1713-1731, 1987.

Tomczak, M., and D.G. Large, Optimum multiparameter analysis of mixing in the themrocline of the eastem Indian Ocean, J. Geophys. Res., 94, 16,141-16,149, 1989.

Tonczak, M., D. G. B. Large, and N. Nancarrow, Identification of diapycnal mixing through optimum multiparameter analysis, 1 . Test of feasibility and sensitivity. J. Geophly's. Res., this issue.

Wannus, K., The form of the temperature-salinity relationship in the thennocline of the western Pacific Ocean, M.Sc. thesis, Mar. Stud. Cent., the Univ. of Sydney, Sydney, N.S.W., Australia.

Zenck, W., B. Klein, and M. Schröder, Cape Verde frontal zone, Deep Sea Res., 38 suppl. 1, 505-530, 1991.

B. Klein, Woods Hole Oceanographic Institution, Woods Hole, MA 025430, USA. (e-mail bklein@whoi.edu)

M. Tomczak, Flinders Institute for Atmospheric and Marine Sciences, Flinders University, GPO Box 2100, Adelaide, South Australia 5001, Australia. (e-mail m.tomczak@es.flinders.edu.au)

(Received April 19, 1993; revised May 9, 1994; accepted July 27, 1994.) 